\title{
Prevalence and Clinical Implication of Microbleeds in Dementia with Lewy Bodies in Comparison with Microbleeds in Alzheimer's Disease
}

\author{
Toshiya Fukui Yoshiyuki Oowan Takahiro Yamazaki Ryuta Kinno \\ Division of Neurology, Internal Medicine, Showa University Northern Yokohama Hospital, \\ Yokohama, Japan
}

\author{
Key Words \\ Dementia with Lewy bodies · Alzheimer's disease · Microbleeds · Periventricular \\ hyperintensities - White matter hyperintensities · Initial symptoms · \\ ${ }^{123}$ I-metaiodobenzylguanidine myocardial scintigraphy
}

\section{Abstract}

Background: Cerebral microbleeds (MBs) have been well investigated in Alzheimer's disease $(A D)$, but not very extensively in non-AD dementias or in dementia with Lewy bodies (DLB). Aims: To elucidate the clinical significance of MBs in DLB. Methods: We compared the prevalence, locations and risk factors for MBs in 59 DLB and 81 AD patients. We visually counted MBs in each of the cortical and subjacent areas (frontal, temporal, parietal and occipital), the basal ganglia and the thalamus, and the brainstem and the cerebellar hemispheres on 1.5-tesla T2*-weighted gradient-recalled-echo MRI images. White matter lesions were semiquantified in fluid-attenuated inversion recovery images according to the Fazekas rating scale. $\boldsymbol{R e}$ sults: While the prevalence of MBs was comparable, MBs tended to be more abundant in DLB than in $A D$ in all brain areas with the exception of the occipital lobes. The number of MBs was positively associated with the severity of white matter lesions but not with other vascular risk factors in either AD or DLB. The presence of MBs could be associated with cognitive impairment at onset. MB-positive DLB patients showed less impairment on ${ }^{123}$ I-metaiodobenzylguanidine myocardial scintigraphy (MIBG scintigraphy) images, supporting the notion of an inverse relationship between vascular lesions and Lewy body pathology. Conclusion: It was suggested that an intricate association between Lewy body pathology, AD-type pathologies and vascular lesions seems to be related to the initial symptoms and results of MIBG scintigraphy in DLB. 


\section{Introduction}

Cerebral microbleeds (MBs) appear as small punctate low-intensity spots that may be best detected in $\mathrm{T} 2 *$-weighted gradient-recalled-echo (fig. 1) and susceptibility-weighted MRI images. In a meta-analysis of five studies that looked at 18 patients, the histological compositions of MBs on MRI were shown to include hemosiderin deposition (49\%), old hematomas (19\%), no specific pathology (15\%), the presence of intact erythrocytes $(13 \%)$, and $1 \%$ each of vascular calcification, microaneurysm and dissected vessels [1]. Thus, more than $80 \%$ of what appears as low-intensity dots on T2*-weighted MRI represents MB-related pathologies, while approximately $20 \%$ may represent nonhemorrhagic changes.

Lipofibrohyalinosis was the primary vascular change generally associated with deepsituated MBs, whereas cerebral amyloid angiopathy (CAA) was associated with cortical MBs especially in patients with dementia [1]. As lipofibrohyalinosis and CAA represent major causes of small-vessel diseases, namely periventricular hyperintensities (PVH), white matter hyperintensities (WMH) and lacunae, the presence of these lesions is considered to be a significant risk factor for MBs [2, 3]. Platelet aggregation inhibitors, but not anticoagulants, were proven to increase the prevalence of MBs especially in the cortical areas [4].

MBs in Alzheimer's disease (AD) have been extensively studied; they are often multiple and found in the cortex, specifically in the occipital lobe [2]. A close association between MBs and small-vessel diseases [1, 3, 5] as well as amyloid pathologies (amyloid plaques and CAA) $[2,5,6]$ has been advocated as the primary cause of the high prevalence of MBs in AD. The prevalence of MBs in AD ranged from 12.5 to 32\%, which is much higher than that in normal controls (3-12\%) [2].

In contrast, the prevalence of MBs in other degenerative dementias has rarely been studied. MBs in patients with frontotemporal lobar degeneration were much less commonly detected even with the use of 7.0-tesla MRI [7]. Reasons for the rareness of MBs were ascribed to a lack of amyloid pathologies and lower average ages in patients with frontotemporal lobar degeneration [7]. From this perspective, the prevalence of MBs in dementia with Lewy bodies (DLB) may be of interest because DLB also has AD-type changes (amyloid pathologies and neurofibrillary tangles). Pathologically, patients with DLB as well as Parkinson's disease with dementia had a significantly higher neuritic Braak stage, cortical amyloid load and capillary CAA than cognitively normal Parkinson's disease patients $[8,9]$. The importance of CAA and concomitant senile plaques/neurofibrillary tangles in developing cognitive impairment in DLB and Parkinson's disease with dementia has been underlined [9].

The coexistence of Lewy bodies (LBs) and AD-type pathologies is common in DLB. Pathological studies have shown that combined pathologies of DLB and AD were found in 15\% [10] up to as much as 76\% [11] of autopsied DLB patients. Further, the amount of LBs correlated positively with AD-type pathologies, while LBs were inversely correlated with vascularrelated pathologies (e.g., atherosclerosis, infarcts and small-vessel disease) [12]. Thus, the prevalence of MBs as well as clinical symptoms in DLB may depend on the subtle balance between DLB pathology (LBs), AD-type pathologies and various vascular factors.

If the occurrence of MBs is dependent primarily on the presence of amyloid pathologies (plaques and CAA) as well as small-vessel diseases and vascular risk factors, but independent of specific proteins that induce neurodegeneration (e.g., $\alpha$-synuclein or tau) [13], it may be hypothesized that the prevalence and locations of MBs in DLB resemble that of AD if concomitant vascular risk factors are similar. However, an autopsy study that compared the prevalence of cortical MBs in $45 \mathrm{AD}$ patients and $8 \mathrm{DLB}$ patients reported that MBs were equally distributed in both diseases but significantly more frequent in AD brains [10].

Given the emerging knowledge on the pathophysiological background of MBs, some unsolved issues with regard to MBs in DLB still remain. First, the clinical prevalence of MBs 


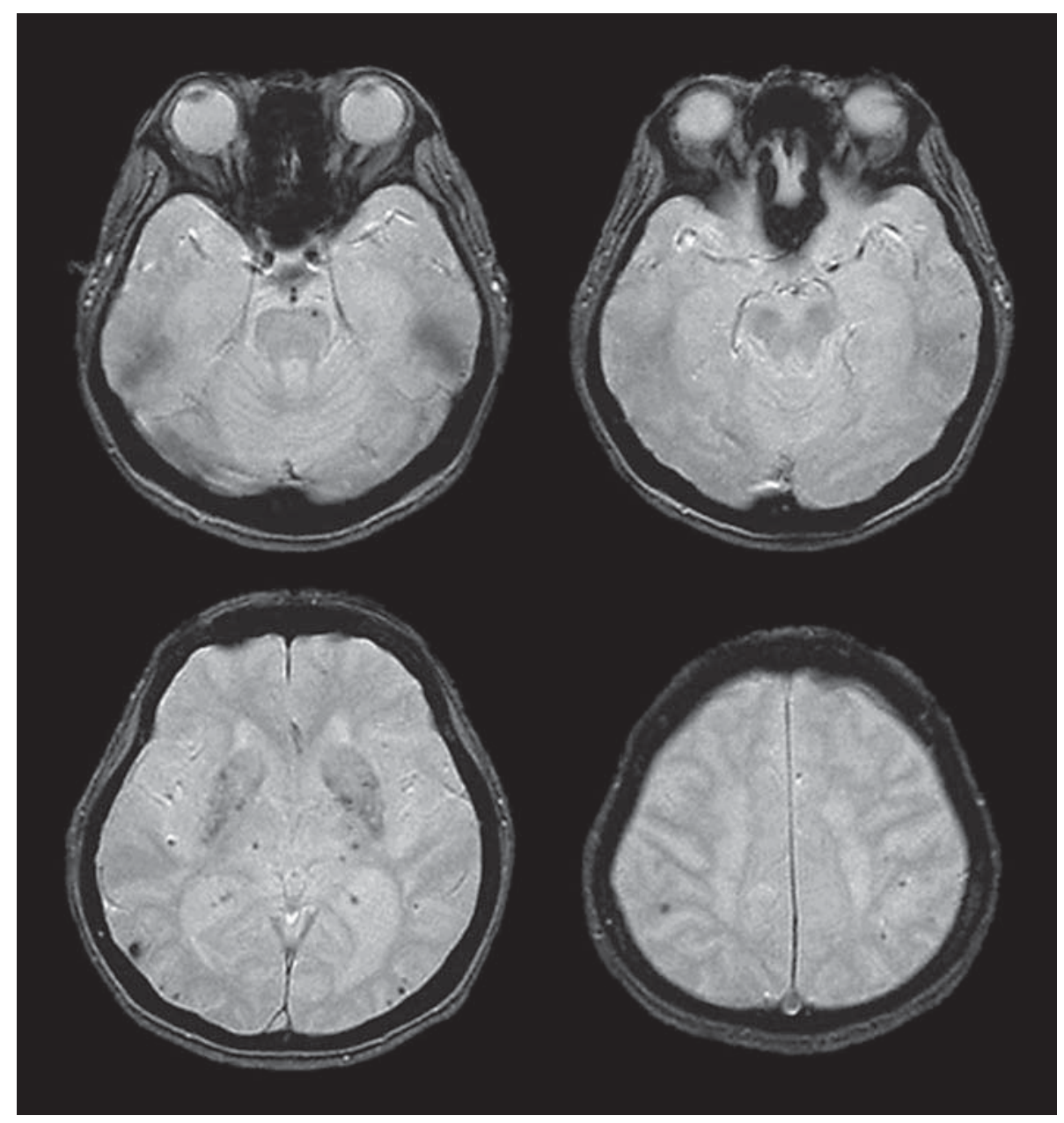

Fig. 1. Representative T2*-weighted gradient-recalled-echo images of MBs from an 81-year-old female with DLB. The patient initially complained about forgetfulness, depressive mood and mild gait disturbance. Early $\mathrm{H} / \mathrm{M}$, late $\mathrm{H} / \mathrm{M}$ and washout ratios on MIBG scintigraphy were 1.82, 1.88 and 39.2\%, respectively. Note multiple small low-intensity black dots on the left side of the pons, temporal and parietal cortices, and basal ganglia and thalami.

in DLB should be elucidated specifically in comparison with that in AD considering shared amyloid pathologies in AD and in DLB. Second, a possible influence of MBs on clinical features of DLB should be clarified. The inverse relationship between LBs and vascular pathology, namely more MBs and less LBs, may obscure the characteristic symptoms of DLB and render the differential diagnoses challenging. Lastly, we were interested in how MBs interact with the results of ${ }^{123}$ I-metaiodobenzylguanidine myocardial scintigraphy (MIBG scintigraphy), an issue that has not yet been addressed. Abnormalities on MIBG scintigraphy [decreased ratios of heart/mediastinum (H/M) uptake and increased washout ratios of MIBG], being a biomarker of DLB pathology, may represent the extent of impaired cardiac sympathetic nerve function induced by LB deposition at presynaptic nerve terminals. The comparison of the severity of MBs and the impairment shown by MIBG scintigraphy may shed some light on the association of vascular lesions (MBs) with LB burden, at least in the myocardium.

We conducted the present study to test the following hypotheses: (1) the prevalence of MBs is similar in AD and DLB patients when vascular loads are comparable, because both 
Fukui et al:: Prevalence and Clinical Implication of Microbleeds in Dementia with Lewy Bodies in Comparison with Microbleeds in Alzheimer's Disease

diseases share amyloid-related pathologies, (2) MB-positive DLB patients may present clinical features which are less typical of DLB than those of MB-negative DLB patients, and (3) the results of MIBG scintigraphy suggest that MB-positive DLB patients may be less impaired, thus reflecting the inverse association between vascular and LB pathologies [12].

\section{Methods}

\section{Patients}

We studied 81 outpatients with AD (38 men, 43 women, average age \pm standard deviation: $79.5 \pm 5.4$ years) and 59 outpatients with DLB (25 men, 34 women, average age: 78.1 \pm 6.0 years). All patients were ambulatory, did not suffer from debilitating illnesses, and had no history of apparent ischemic diseases (cerebral infarction, transient ischemic attack, coronary arterial disease or peripheral arteriosclerotic occlusive disorders) and/or ischemic electrocardiographic changes. The diagnosis of $\mathrm{AD}$ was made with the criteria from the National Institute of Neurological and Communicative Disorders and Stroke and the Alzheimer's Disease and Related Disorders Association (NINCDS-ADRDA) [14], and the diagnosis of DLB was made with the consensus criteria [15]. We utilized brain MRI, SPECT and MIBG scintigraphy as adjunctive measures in the differentiation of AD and DLB. We assessed cognitive levels at the time of diagnosis using the revised Hasegawa Dementia Scale (HDSR), a short cognitive test consisting of tasks concerning orientation, verbal and visual memory, calculation and categorical word fluency with a full score of 30 [16]. Since the HDSR scores ranged between 29 and 5 (median 19), the severity of dementia corresponded to mild to severe, but mostly to moderate.

The study was approved by the Ethics Committee of the Showa University Northern Yokohama Hospital. The patients and/or their caregivers provided written consent to participate in the study after the procedures had been explained in detail.

\section{Vascular Risk Factors and Vascular Lesions on MRI}

We considered the presence of and/or the treatment for arterial hypertension (high blood pressure, HBP), diabetes mellitus (DM), dyslipidemia (Dyslipid) and atrial fibrillation (Af) at the time of evaluation as vascular risk factors. All patients underwent 1.5-tesla MRI examinations as a routine diagnostic procedure, and fluid-attenuated inversion recovery and T2*-weighted gradient-recalled-echo images were obtained. We used the same MRI equipment for all participants with a fixed slice thickness, gap width and other related parameters to avoid technical errors. Small-vessel lesions represented by PVH and WMH were semiquantified on fluid-attenuated inversion recovery images according to the Fazekas method [17]. Lacunae, if any, were considered as representing small-vessel disease and were semiquantified together with WMH. MBs were visually counted on T2* images in the cortical and subjacent areas in the frontal, temporal, parietal and occipital lobes (cortical), thalamus and basal ganglia (deep), and brainstem and cerebellar hemispheres (infratentorial). Lowintensity spots located in the pallidum were excluded because they are highly likely to represent calcium deposition.

\section{Initial Symptoms of DLB and Vascular Factors}

In a previous study, we found that patients with DLB may present diverse symptoms at onset including cognitive, psychiatric or physical/autonomic ones, alone or in various combinations [18]. Accordingly, we categorized the initial symptoms of DLB into three realms: cognitive (symptoms ascribed to impairment of memory, executive function, visuospatial 
Fukui et al.: Prevalence and Clinical Implication of Microbleeds in Dementia with Lewy Bodies in Comparison with Microbleeds in Alzheimer's Disease

abilities and others), psychiatric [apathy, depression, delusions, hallucinations, realistic but pseudo awareness of someone behind oneself (die leibhaftige Bewusstheit) and phantom boarder] and physical (parkinsonism and various autonomic disturbances such as orthostatic hypotension).

We first compared the prevalence of each of the initial symptoms in MB-positive and MB-negative DLB patients. We then looked for those factors that might determine the type of the initial symptoms such as the age of the patients, their vascular risks (HBP, DM, Dyslipid and Af) as well as their vascular lesions (PVH, WMH and total MB counts) using logistic regression analysis.

\section{MIBG Scintigraphy in DLB and Vascular Factors}

In order to confirm the clinical diagnosis, MIBG scintigraphy was conducted in patients who satisfied the criteria for DLB. However, MIBG scintigraphy was not available for 8 DLB patients for various social reasons. We obtained early (20 min after injection) and late ( $4 \mathrm{~h}$ after injection) H/M uptake ratios as well as a washout ratio of MIBG. Because myocardial damage can arise from a variety of other causes, and this is a major confounding factor in the interpretation of MIBG scintigraphy, patients with an apparent history of or showing electrocardiographic changes suggestive of ischemic heart diseases were excluded in the recruitment stage. Diabetic autonomic neuropathy may also affect the results of MIBG scintigraphy [19]. However, we included 17 DLB patients with DM as well, because the presence of DM did not adversely affect early H/M (DM $1.71 \pm 0.21$, non-DM $1.58 \pm 0.26, \mathrm{p}=0.07)$, late H/M (DM $1.63 \pm 0.31$, non-DM $1.57 \pm 0.39, \mathrm{p}=0.38$ ) or washout ratios (DM $41.3 \pm 7.8 \%$, non-DM 39.8 $\pm 7.4 \%, \mathrm{p}=0.45$ ). First, we compared the early and late $\mathrm{H} / \mathrm{M}$ ratios and washout ratios of MIBG between MB-positive and MB-negative patients. Then we used linear regression analyses to investigate whether the results of MIBG scintigraphy were affected by the age of the patients and the presence and severity of vascular risk factors and lesions.

\section{Statistics}

Because the normality of data distribution was unknown, we used the Mann-Whitney test for numerical comparisons and the $\chi^{2}$ test for categorical comparisons. The linear regression analyses looked for factors related to the total number of MBs which might influence the clinical diagnosis (AD or DLB), age, vascular risk factors (HBP, DM, Dyslipid and Af), the use of antiplatelet agents and ischemic vascular lesions (Fazekas scores for PVH and WMH). The contribution of the age of the patients, vascular risks (HBP, DM, Dyslipid and Af) and vascular lesions (PVH, WMH and total MB counts) to each initial symptom (cognitive, psychiatric and physical) was investigated by stepwise logistic analyses. Finally, the influence of the above-mentioned demographic and vascular factors on the results of MIBG scintigraphy in DLB patients was assessed by stepwise linear regression analyses. $p<0.05$ was considered statistically significant. Statistical analyses were conducted by SPSS Version 17.0 (Japanese version).

\section{Results}

Patient Demography (fig. 2)

Between the AD and DLB patients, there were no statistical differences in the age of the patients (AD $79.5 \pm 5.4$ years, DLB $78.1 \pm 6.0$ years, $p=0.11$ ), the proportion of men (AD 47\%, DLB $42 \%, p=0.36$ ), the HDSR scores (AD 18.6 \pm 5.6 , DLB 17.2 $\pm 7.1, p=0.35$ ), the prevalence of HBP (AD 59\%, DLB 49\%, p = 0.16), DM (AD 32\%, DLB 31\%, p = 0.49), Af (AD 6\%, DLB 0\%, $p=0.11$ ), the use of antiplatelet agents (AD 15\%, DLB 7\%, $p=0.11$ ) and the Fazekas scores 
Fukui et al.: Prevalence and Clinical Implication of Microbleeds in Dementia with Lewy Bodies in Comparison with Microbleeds in Alzheimer's Disease

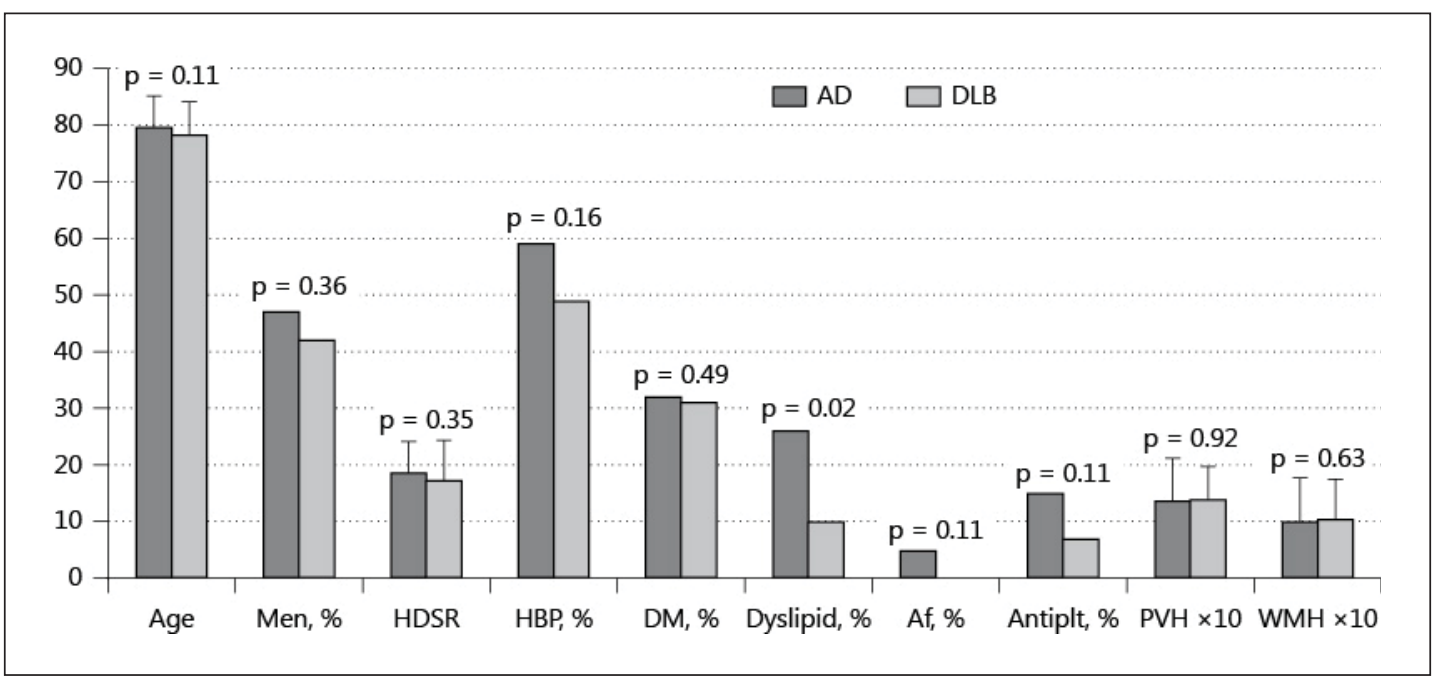

Fig. 2. Patient demography of subjects with $A D$ and DLB. No differences existed between these two disease groups in terms of average age, scores for the HDSR, vascular risk factors including HBP, DM, Af and use of antiplatelet agents (Antiplt) and Fazekas scores for PVH and WMH. Dyslipid was significantly more frequent in AD than DLB.

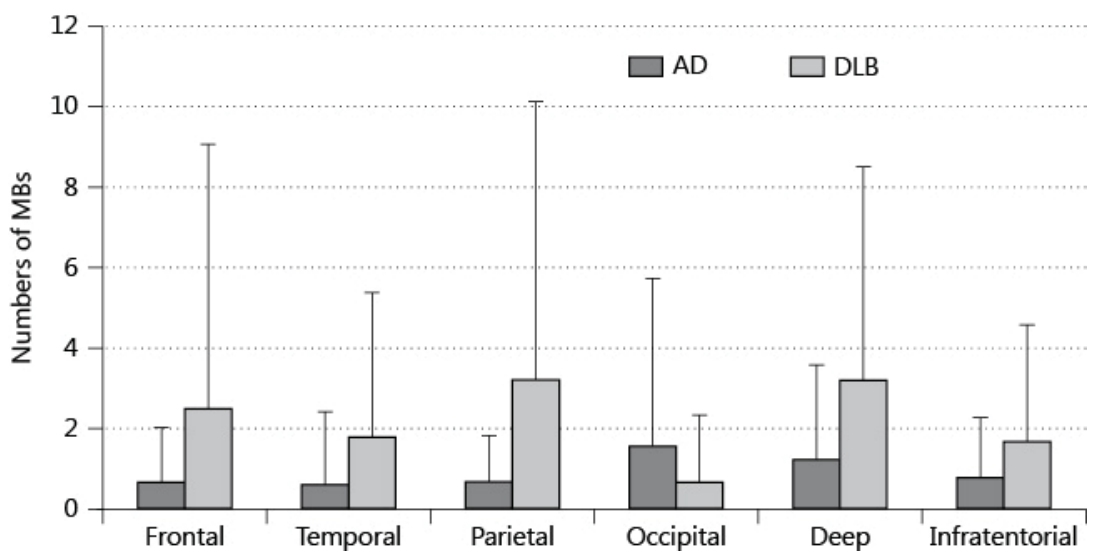

Fig. 3. Numbers of counted MBs in each brain area. MB counts were nonsignificantly higher in DLB than in $\mathrm{AD}$ in all brain areas except the occipital lobes.

for PVH (AD 1.36 \pm 0.75 , DLB $1.39 \pm 0.59, \mathrm{p}=0.92$ ) and WMH (AD $1.00 \pm 0.77$, DLB $1.05 \pm 0.71$, $\mathrm{p}=0.63)$. Among vascular risk factors, Dyslipid was significantly more frequent in the AD than in the DLB patients (AD 26\%, DLB 10\%, $\mathrm{p}=0.02$ ).

\section{Prevalence of $M B$ s in $A D$ and $D L B$}

The prevalence of total MBs was 19.8\% (16 out of 81 patients) for AD and 16.9\% (10 out of 59 patients) for DLB, and we found no statistical difference between the two patient groups $(p=0.42)$. However, the total MB counts were higher in DLB $(2.2 \pm 9.6)$ than in AD (1.1 \pm 4.3$)$, although not significantly so $(p=0.71$ ). Local MB counts (fig. 3 ) were also larger in all brain 
Fukui et al:: Prevalence and Clinical Implication of Microbleeds in Dementia with Lewy Bodies in Comparison with Microbleeds in Alzheimer's Disease

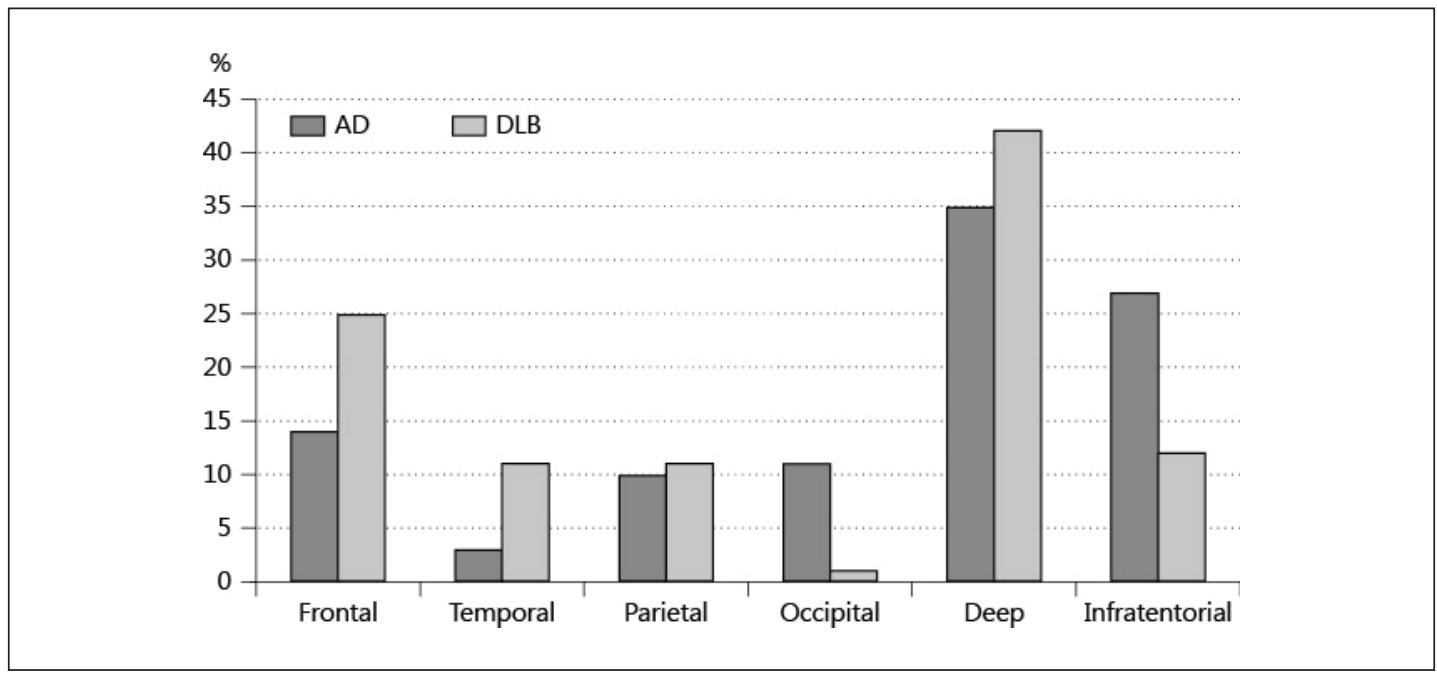

Fig. 4. Distribution of MBs. The proportion of local MBs as the percentage of total MBs was comparable in $\mathrm{AD}$ and DLB. MBs in DLB were frequently located in the frontal and deep regions but rarely in the occipital areas, although not significantly so.

areas in DLB (frontal: AD $0.7 \pm 1.4$, DLB $2.5 \pm 6.6$; temporal: AD $0.6 \pm 1.8$, DLB $1.8 \pm 3.6$; parietal: AD $0.7 \pm 1.1$, DLB $3.2 \pm 6.9$; deep: AD $1.3 \pm 2.3$, DLB $3.2 \pm 5.3$; infratentorial: AD 0.8 \pm 1.5 , DLB $1.7 \pm 2.9$ ) with the exception of the occipital lobes where MBs were more numerous in $\mathrm{AD}$ ( $\mathrm{AD} 1.6 \pm 4.2$, DLB $0.7 \pm 1.6$ ). Because of the small numbers of MB-positive patients in each group (AD 16, DLB 10) and a large variability (standard deviation) among the patients, the differences between $\mathrm{AD}$ and DLB did not reach statistical significance.

The distribution of MBs, described as the proportion of the local MBs as the percentage of the total MBs, was also comparable in AD and DLB (fig. 4). In DLB, MBs were rare in the occipital areas (DLB 1\%, AD 11\%, p = 0.41) and were frequently located in the frontal (DLB $25 \%, \mathrm{AD} 14 \%, \mathrm{p}=0.62$ ) and deep areas (DLB 42\%, AD 35\%, $\mathrm{p}=0.51$ ). However, the differences between $\mathrm{AD}$ and $\mathrm{DLB}$ did not reach statistical significance in any region.

Patient Backgrounds and Vascular Factors in Patients with and without MBs (table 1)

We then compared patient demography, vascular risk factors and PVH/WMH between the patients with and without MBs in AD and DLB, respectively. The age of the patients, their gender distribution and the scores of the HDSR were comparable in AD. In contrast, the MB-positive DLB patients were significantly older $(p=0.04)$ and tended to score better on the HDSR $(\mathrm{p}=0.10)$ than the MB-negative DLB patients. Their backgrounds with regard to vascular risks/lesions were similar in AD and DLB. The PVH and WMH scores were significantly higher, and HBP and the use of antiplatelet agents were more frequent, although not significantly so, in the MB-positive patients, and this suggests a close association between major vascular risks/lesions and MBs. In contrast, the frequency of DM and Dyslipid was lower in the MB-positive patients.

We went on to conduct a stepwise linear regression analysis to determine the most relevant factor(s) of the total counts of MBs in the whole cohort of AD and DLB patients. Independent variables included the type of diseases (AD or DLB), age, gender, vascular risk factors (HBP, DM, Dyslipid and Af), the use of antiplatelet agents, and scores for PVH and WMH. We found that the severity of WMH was a significant accelerating factor of the total numbers of 
Fukui et al.: Prevalence and Clinical Implication of Microbleeds in Dementia with Lewy Bodies in Comparison with Microbleeds in Alzheimer's Disease

Table 1. Patient demography, vascular risk factors and Fazekas scores in MB-positive and MB-negative patients with AD or DLB (see text for discussion)

\begin{tabular}{llllllllllll}
\hline & $\mathrm{n}$ & $\begin{array}{l}\text { Age } \\
\text { years }\end{array}$ & $\begin{array}{l}\text { Men } \\
\%\end{array}$ & HDSR & $\begin{array}{l}\text { HBP } \\
\%\end{array}$ & $\begin{array}{l}\text { DM } \\
\%\end{array}$ & $\begin{array}{l}\text { Dyslipid } \\
\%\end{array}$ & $\begin{array}{l}\text { Af } \\
\%\end{array}$ & $\begin{array}{l}\text { Plt } \\
\%\end{array}$ & PVH & WMH \\
\hline $\mathrm{MB}(+)$ AD & 16 & $78.4 \pm 5.3$ & 56 & $18.1 \pm 6.9$ & 69 & 25 & 19 & 6 & 25 & $1.75 \pm 0.86$ & $1.56 \pm 0.81$ \\
$\mathrm{MB}(-)$ AD & 65 & $79.8 \pm 5.4$ & 45 & $18.8 \pm 5.3$ & 57 & 34 & 28 & 5 & 12 & $1.26 \pm 0.69$ & $0.86 \pm 0.70$ \\
$\mathrm{p}$ & & 0.29 & 0.29 & 0.85 & 0.29 & 0.36 & 0.35 & 0.59 & 0.18 & 0.03 & 0.002 \\
\hline $\mathrm{MB}(+) \mathrm{DLB}$ & 10 & $81.0 \pm 3.3$ & 60 & $20.6 \pm 5.2$ & 70 & 10 & 0 & 0 & 20 & $1.80 \pm 0.42$ & $1.50 \pm 0.85$ \\
$\mathrm{MB}(-) \mathrm{DLB}$ & 49 & $77.5 \pm 6.2$ & 39 & $16.5 \pm 7.2$ & 45 & 35 & 12 & 0 & 4 & $1.31 \pm 0.59$ & $0.96 \pm 0.64$ \\
$\mathrm{p}$ & & 0.04 & 0.19 & 0.10 & 0.14 & 0.12 & 0.31 & - & 0.13 & 0.005 & 0.03 \\
\hline
\end{tabular}

Plt = Antiplatelet agents.

MBs $\left(\beta=0.28, p=0.01, R^{2}=0.28\right)$. Other factors exerted no significant influence on the numbers of MBs.

\section{Effects of MBs on the Initial Symptoms in DLB Patients (fig. 5)}

At onset, the MB-positive DLB patients had a tendency to present cognitive symptoms more frequently ( $90 \%$ of MB-positive patients, $p=0.045$ ) and psychiatric symptoms less frequently $(20 \%, \mathrm{p}=0.051$ ) than the MB-negative patients (cognitive $56 \%$, psychiatric $54 \%$ ). MB-positive DLB patients showed no physical impairment at onset, while 19\% of the MB-negative patients presented physical symptoms as the initial symptoms. Because of a small number of MB-positive patients $(n=10)$, the intragroup difference among the prevalence of cognitive, psychiatric and physical impairments did not reach statistical significance $(\mathrm{p}=0.20)$. The MB-negative patients tended to show cognitive, psychiatric and physical symptoms more evenly than the MB-positive patients.

Stepwise logistic regression analyses, with age, gender, vascular risk factors, $\mathrm{PVH} / \mathrm{WMH}$ scores and MB counts as independent variables, appeared to show that the presence of HBP was a significant factor leading to cognitive impairment as the initial symptom of DLB [odds ratio $\left.(O R)=4.2, p=0.01, R^{2}=0.11\right]$, that the severity of $P V H$ was a significant factor leading to psychiatric disorders as the initial symptoms $\left(\mathrm{OR}=0.24, \mathrm{p}=0.004, \mathrm{R}^{2}=0.13\right.$ ), and that the age of the patients was a significant factor leading to physical problems as the initial symptoms $\left(\mathrm{OR}=0.84, \mathrm{p}=0.004, \mathrm{R}^{2}=0.12\right)$. While the presence of HBP raised the likelihood of cognitive symptoms at onset, lower, rather than higher, PVH scores and a lower age of the patient (ORs $=0.24$ and 0.84 are smaller than 1.0 ) raised the likelihood of psychiatric and physical symptoms at onset, respectively. The number of MBs was not an independent factor influencing the initial symptoms of DLB.

Association of MBs with the Results of MIBG Scintigraphy in DLB Patients (fig. 6)

MIBG scintigraphy was available in 53 DLB patients. First, we compared the results of MIBG scintigraphy between 10 MB-positive and 49 MB-negative DLB patients. There was a tendency that the early H/M ratios were lower (more impaired) in the MB-negative $1.59 \pm$ $0.26)$ than in the MB-positive $(1.78 \pm 0.12)$ patients $(p=0.06)$. The late $H / M$ ratios were lower in the MB-negative $(1.57 \pm 0.38)$ than in the MB-positive patients $(1.77 \pm 0.21)$, but the difference did not reach statistical significance $(p=0.24)$. Washout ratios were abnormally enhanced regardless of the presence of MBs (39.9\% for MB-positive and 40.4\% for MB-negative patients, $p=0.71$ ). 
Fig. 5. Association between MBs and initial symptoms. MB-positive DLB patients tended to present cognitive symptoms at onset more frequently than psychiatric or physical symptoms. MB-negative patients tended to have a more even distribution of cognitive, psychiatric and motor symptoms.

Fig. 6. Association between MBs and results of MIBG scintigraphy. Both early and late $\mathrm{H} / \mathrm{M}$ ratios were lower (more impaired) in MB-negative than in MB-positive patients. The differences in the early $\mathrm{H} / \mathrm{M}$ ratios reached statistical significance. Washout ratios were abnormally enhanced regardless of the presence of MBs. Washout $\mathrm{R} / 10$ = Washout ratios divided by 10 .

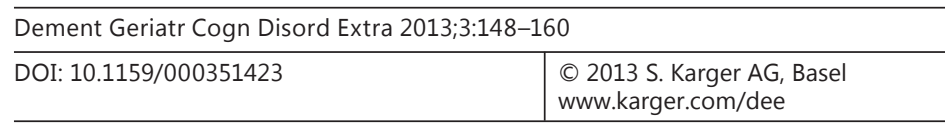

Fukui et al.: Prevalence and Clinical Implication of Microbleeds in Dementia with Lewy Bodies in Comparison with Microbleeds in Alzheimer's Disease
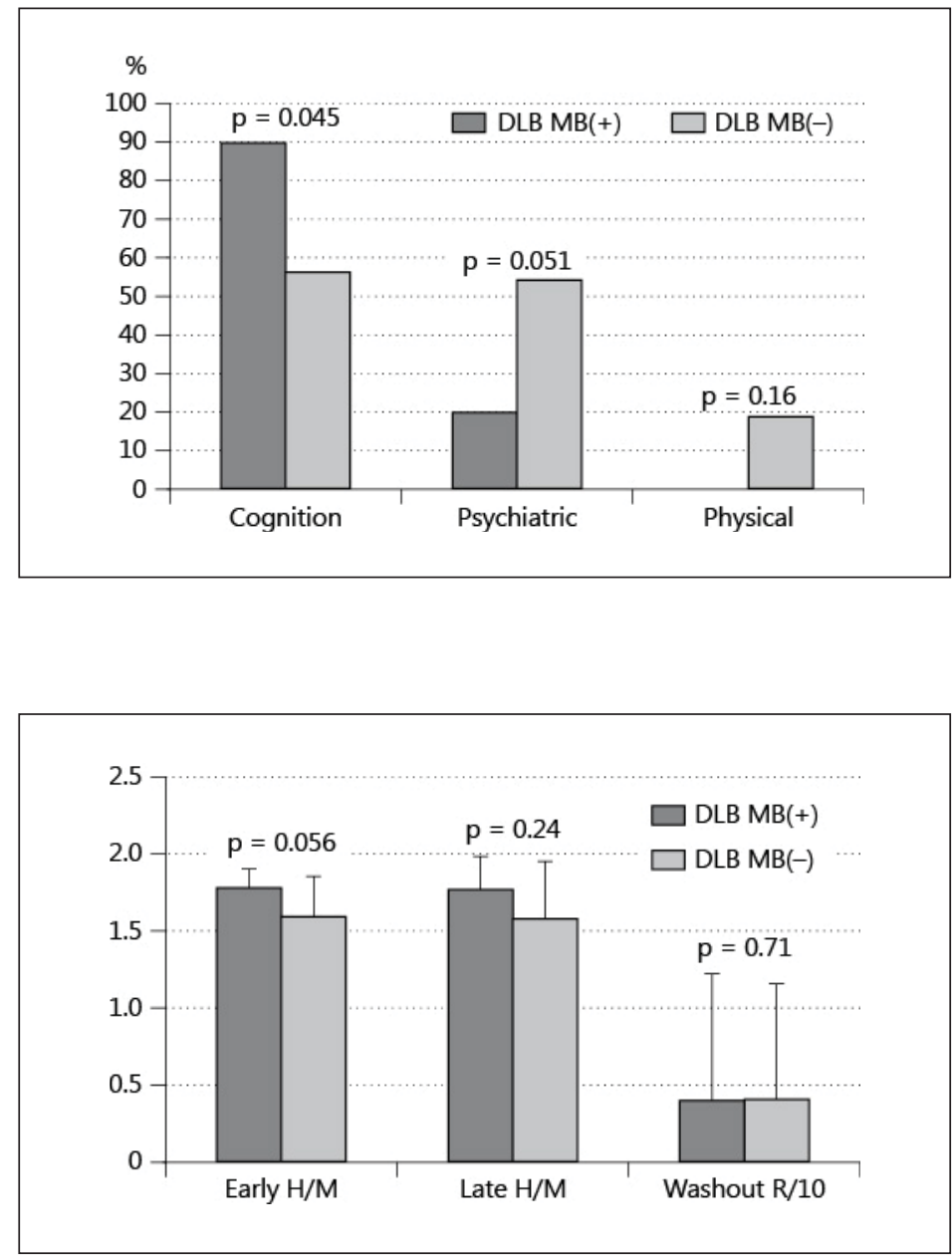

In a stepwise linear regression model with age and gender distribution of patients, each vascular risk factor, $\mathrm{PVH} / \mathrm{WMH}$ and the total $\mathrm{MB}$ counts as independent variables, the presence of Dyslipid $\left(\beta=-0.35, R^{2}=0.12, p=0.01\right.$ ) significantly lowered (worsened) early H/M ratios. PVH $(\beta=0.26, p=0.06)$ and DM $(\beta=0.24, p=0.08)$ showed a tendency to contribute to higher (better) early $\mathrm{H} / \mathrm{M}$ ratios. Other factors, including MB counts, were not independent factors influencing the results of early $\mathrm{H} / \mathrm{M}$ ratios. No factor was associated with either late $\mathrm{H} / \mathrm{M}$ ratios or washout ratios.

\section{Discussion}

This study clarified several clinical implications of MBs in DLB. The prevalence of MBs in $\mathrm{AD}$ and DLB patients was comparable, while the actual number of MBs was larger in DLB. The distribution of MBs was similar in AD and DLB with the exception of the occipital dominancy in $\mathrm{AD}$ that was not observed in DLB. The Fazekas scores for PVH and WMH were higher in the MB-positive patients, and the severity of WMH was the significant and exclusive determinant of the total number of MBs in both AD and DLB. Conversely, the type of disease (AD or DLB) was not a factor influencing the number of MBs. 
We found that DM and Dyslipid were less frequent in MB-positive patients. A lower frequency of Dyslipid in MB-positive patients may be in line with a general understanding that a low cholesterol level is one of the risks of cerebral hemorrhage and MBs [20]. However, we could not find reasonable accounts for the lower prevalence of DM in these patients, given that DM is a well-known major cause of small-vessel diseases and an accelerating factor of amyloid deposition [21]. This issue needs to be addressed in future studies.

Initially, we hypothesized that AD and DLB patients may have a similar prevalence and severity of MBs because of common amyloid pathologies. The present study showed that both $\mathrm{AD}$ and DLB patients had essentially the same prevalence of total as well as local MBs, possessing similar backgrounds in terms of patient demography and vascular risk factors. PVH and WMH were more prominent in MB-positive AD and DLB patients. Viewed from this perspective, we assumed that the occurrence and the number of MBs in both AD and DLB were dependent mainly on the presence and severity of vascular risk factors and small-vessel diseases including amyloid deposition.

As a proof that amyloid pathologies are involved in the pathogenesis of MBs in AD, MBs were more numerous in the occipital areas in AD where CAA predominates [2]. However, DLB patients did not show an occipital dominance in MB distribution. It may be just a bias derived from a small sample size $(n=10)$. Alternatively, the present results may suggest a possible difference in the distribution of CAA between AD and DLB. Previous pathological studies showed that CAA in DLB and Parkinson's disease with dementia may be accentuated either in the occipital areas or generalized brain areas including the frontal lobes $[8,9,12]$. Future pathological studies should focus on the topographical association between MBs and CAA in DLB.

In other brain areas, MBs were more abundant in DLB patients than in AD patients, although not significantly so. These results may not agree with the autopsy findings that MBs were significantly more frequent in $\mathrm{AD}$ brains, while the distribution of MBs was equal in $\mathrm{AD}$ and DLB brains [10]. This discordance is likely due to the differences in methodology (MRI vs. pathological); MRI studies may offer more advantages than a pathological approach in that the whole brain can be scanned in search for MBs.

The reasons for higher MB counts in DLB patients than in AD patients are not clear, because we found no differences in the prevalence of vascular risk factors and the severity of $\mathrm{PVH} / \mathrm{WMH}$ between AD and DLB. It may be possible that DLB patients are more prone to develop multiple MBs or that they had a higher proportion of nonhemorrhagic low-intensity spots on MRI. Again, this issue awaits further studies to clarify pathological entities of MBs on MRI in patients with DLB.

The next hypotheses suggested that clinical features and the results of MIBG scintigraphy in DLB patients are subject to the influences of MBs. These hypotheses were based on previous studies showing that, given the same clinical severity, in DLB patients with vascular burden the LB pathology may be less abundant than in DLB patients without vascular lesions [12].

As to the factors influencing the initial symptoms of DLB, a significant association was found between the higher prevalence of HBP and cognitive impairment, milder PVH and psychiatric disorders, and lower age and physical symptoms. The association between HBP and cognitive impairment has been well established in previous studies, even in mild cognitive impairment [22]. We found that less severe PVH raised the likelihood that psychiatric symptoms led to the initial presentation, although frontal deep WMH are reportedly associated with depression in both AD and DLB patients [23]. We need prudent interpretations of these conflicting results: one possible explanation may be that milder PVH signifies a more abundant LB pathology [12], which may in turn induce characteristic psychiatric symptoms of DLB such as hallucinations, delusions and cognitive fluctuations. Physical symptoms at onset were associated with younger age. This result may be to some extent associated with 
the finding that the pure form of DLB that lacks amyloid pathologies tends to present initial symptoms of motor impairments (parkinsonism) at lower ages [11].

Conversely, the presence of MBs per se was not an independent factor of which symptoms appear at the onset of DLB, although MB-positive DLB patients were more likely to initially present cognitive symptoms than MB-negative patients. It may be the case that the presence of MBs implies more abundant AD-type pathologies and thus more prominent cognitive impairment at onset. On the other hand, higher scores on the HDSR at the time of diagnosis in MB-positive patients may not contradict the tendency of these patients to present cognitive impairment at onset because of the differences in the time of assessment (at diagnosis vs. at onset) and the qualitative contents of cognitive impairment: the HDSR, which is a memoryand language-dependent test, may not necessarily reflect executive or visuospatial impairments.

Finally, we compared the total numbers of MBs and the results of MIBG scintigraphy. We found that the impairment visible on MIBG scintigraphy, especially the early H/M ratios, was less severe in MB-positive patients. Furthermore, regression models showed a significant association of Dyslipid and a trend toward significance for PVH and DM with early H/M ratios.

The presence of Dyslipid, mainly hypercholesterolemia, was independently associated with lower (more impaired) early H/M ratios in a linear regression model. Although Dyslipid was more prevalent in the MB-negative group where early $\mathrm{H} / \mathrm{M}$ ratios were lower, this result from a regression analysis is unlikely to be a consequence of multicollinearity. Dyslipid might induce subclinical arteriosclerosis and myocardial damage that possibly prevent MIBG uptake. In any case, the implication of this result remains uncertain until further investigations.

On the other hand, the presence of PVH and DM appeared to counteract the impairment of the myocardial sympathetic system caused by LB deposition. In Parkinson's disease, abnormalities on MIBG scintigraphy correlate roughly with the clinical severity of the disease [24], suggesting that the LB burden may correlate with an impaired uptake of MIBG in the myocardium. Given that the same is true in DLB, we assume that the presence of PVH and DM, signifying more severe small-vessel diseases, may mean a smaller LB burden and thus milder impairment visible on MIBG scintigraphy.

What is the clinical implication of this inverse association? As can be inferred from the observation that vascular lesions are a significant inducing factor of overt dementia in patients with a subclinical AD pathology [25], first it is likely that vascular lesions that complicate LB pathology may accelerate the emergence of clinical DLB symptoms, and this would make a diagnosis of DLB possible at an earlier stage. Second, MB-positive DLB patients, with heavier vascular and $\mathrm{AD}$-type pathologies but less LBs, may present a rather atypical clinical course and symptoms when compared to MB-negative patients with a more profuse LB pathology. Based on these differences between MB-positive and MB-negative patients, MB-positive DLB patients may be pertinently described as a DLB variant of AD with cerebrovascular disease. Future studies should investigate the intricate association among LB pathologies, vascular lesions and AD-type pathologies, and their composite effects on the initial symptoms and results obtained using MIBG scintigraphy from both clinical and pathological perspectives.

This study has several limitations. As mentioned repeatedly, the major limitation is a lack of pathological confirmation of the clinical diagnosis of AD and DLB as well as the composition of low-intensity spots visually judged to be MBs on T2*-weighted MRI, $20 \%$ of which may represent nonhemorrhagic changes [1]. At the time of writing this report, all the participants are physically well and continue to be treated at the outpatient department, making pathological investigations impossible. As to the differential diagnosis of AD and DLB, we took extra care to detect the presence of extrapyramidal signs, early hallucinations, delusions and cognitive fluctuations. As adjunctive measures of a differential diagnosis, we conducted a 
Fukui et al.: Prevalence and Clinical Implication of Microbleeds in Dementia with Lewy Bodies in Comparison with Microbleeds in Alzheimer's Disease

SPECT study in all subjects and MIBG scintigraphy in 9 patients finally diagnosed with AD, in whom there was still a possibility of DLB. Further, we measured $\beta$-amyloid and tau in the cerebrospinal fluid in 3 clinically atypical patients, who turned out to have AD pathologies according to these biomarkers. We believe that these measures may have partially compensated for the lack of pathological data. Second, a more sensitive detection of MBs would have been possible with MRI scanning in a higher magnetic field or susceptibility-weighted images than with $\mathrm{T} 2 *$-weighted images in a 1.5-tesla magnetic field, neither of which was available in this study. Third, MIBG scintigraphy was not available in 8 out of 59 DLB patients because of the patient's refusal, the nonavailability of accompanying caregivers, the failure to appear for an examination or other reasons.

In spite of these limitations, this study may help understand the prevalence, the association with vascular factors and the clinical implications of MBs in DLB, specifically in comparison with MBs in AD. Future studies are warranted to investigate those issues which have remained unanswered in the present study, namely the composition of MBs on MRI in DLB patients, the pathologically defined distribution of MBs in AD and DLB especially with regard to the distribution in the occipital lobes, the quantitative association among smallvessel lesions ( $\mathrm{PVH}, \mathrm{WMH}$ and MBs), AD-type pathologies and $\mathrm{LB}$ pathology in the brain as well the myocardium.

\section{Acknowledgment}

This study was partially supported by a research grant from Eisai Co.

\section{References}

1 Shoamanesh A, Kwok CS, Benavente 0: Cerebral microbleeds: histopathological correlation of neuroimaging. Cerebrovasc Dis 2011;32:528-534.

-2 Pettersen JA, Sathiyamoorthy G, Gao FQ, Szilagyi G, Nadkarni NK, St George-Hyslop P, Rogaeva E, Black SE: Microbleed topography, leukoaraiosis, and cognition in probable Alzheimer disease from the Sunnybrook Dementia Study. Arch Neurol 2008;65:790-795.

-3 Yamada S, Saiki M, Satow T, Fukuda A, Ito M, Minami S, Miyamoto S: Periventricular and deep white matter leukoaraiosis have a closer association with cerebral microbleeds than age. Eur J Neurol 2012;19:98-104.

-4 Vernooij MW, Haag MD, van der Lugt A, Hofman A, Krestin GP, Stricker BH, Breteler MM: Use of antithrombotic drugs and the presence of cerebral microbleeds: the Rotterdam Scan Study. Arch Neurol 2009;66: 714-720.

5 Cordonnier C: Brain microbleeds: more evidence, but still a clinical dilemma. Curr Opin Neurol 2011;24: 69-74.

6 Goos JD, Kester MI, Barkhof F, Klein M, Blankenstein MA, Scheltens P, van der Flier WM: Patients with Alzheimer disease with multiple microbleeds: relation with cerebrospinal fluid biomarkers and cognition. Stroke 2009; 40:3455-3460.

7 De Reuck J, Deramecourt V, Cordonnier C, Auger F, Durieux N, Bordet R, Maurage CA, Leys D, Pasquier F: Detection of microbleeds in post-mortem brains of patients with frontotemporal lobar degeneration: a 7.0-tesla magnetic resonance imaging study with neuropathological correlates. Eur J Neurol 2012;19:13551360 .

$>8$ Jellinger KA, Attems J: Prevalence and impact of vascular and Alzheimer pathologies in Lewy body disease. Acta Neuropathol 2008;115:427-436.

$\longrightarrow 9$ Jellinger KA, Attems J: Cerebral amyloid angiopathy in Lewy body disease. J Neural Transm 2008;115:473482.

10 De Reuck J, Deramecourt V, Cordonnier C, Leys D, Pasquier F, Maurage CA: Prevalence of small cerebral bleeds in patients with a neurodegenerative dementia: a neuropathological study. J Neurol Sci 2011;300:63-66.

11 Kosaka K: Diffuse Lewy body disease in Japan. J Neurol 1990;237:197-204.

12 Ghebremedhin E, Rosenberger A, Rüb U, Vuksic M, Berhe T, Bickeböller H, de Vos RA, Thal DR, Deller T: Inverse relationship between cerebrovascular lesions and severity of Lewy body pathology in patients with Lewy body diseases. J Neuropathol Exp Neurol 2010;69:442-448. 
13 Goos JD, Henneman WJ, Sluimer JD, Vrenken H, Sluimer IC, Barkhof F, Blankenstein MA, Scheltens PH, van der Flier WM: Incidence of cerebral microbleeds: a longitudinal study in a memory clinic population. Neurology 2010;74:1954-1960.

14 McKhann G, Drachman D, Folstein M, Katzman R, Price D, Stadlan EM: Clinical diagnosis of Alzheimer's disease: report of the NINCDS-ADRDA Work Group under the auspices of Department of Health and Human Services Task Force on Alzheimer's Disease. Neurology 1984;34:939-944.

15 McKeith IG, Dickson DW, Lowe J, Emre M, O’Brien JT, Feldman H, Cummings J, Duda JE, Lippa C, Perry EK, Aarsland D, Arai H, Ballard CG, Boeve B, Burn DJ, Costa D, Del Ser T, Dubois B, Galasko D, Gauthier S, Goetz CG, Gomez-Tortosa E, Halliday G, Hansen LA, Hardy J, Iwatsubo T, Kalaria RN, Kaufer D, Kenny RA, Korczyn A, Kosaka K, Lee VM, Lees A, Litvan I, Londos E, Lopez OL, Minoshima S, Mizuno Y, Molina JA, Mukaetova-Ladinska EB, Pasquier F, Perry RH, Schulz JB, Trojanowski JQ, Yamada M, Consortium on DLB: Diagnosis and management of dementia with Lewy bodies: third report of the DLB Consortium. Neurology 2005;65:1863-1872.

-16 Hosokawa T, Yamada Y, Isagoda A, Nakamura R: Psychometric equivalence of the Hasegawa Dementia ScaleRevised with the Mini-Mental State Examination in stroke patients. Percept Mot Skills 1994;79:664-666.

17 Fazekas F, Chawluk JB, Alavi A, Hurtig HI, Zimmerman RA: MR signal abnormalities at 1.5 T in Alzheimer's dementia and normal aging. AJR Am J Roentgenol 1987;149:351-356.

-18 Fukui T, Hori K, Yoshimasu H: Onset patterns and initial symptoms of dementia with Lewy bodies: possible pathophysiological diversities deduced from a SPECT study. Dement Geriatr Cogn Disord Extra 2011;1:237248.

19 Scholte AJ, Schuijf JD, Delgado V, Kok JA, Bus MT, Maan AC, Stokkel MP, Kharagitsingh AV, Dibbets-Schneider $\mathrm{P}$, van der Wall EE, Bax JJ: Cardiac autonomic neuropathy in patients with diabetes and no symptoms of coronary artery disease: comparison of ${ }^{123} \mathrm{I}$-metaiodobenzylguanidine myocardial scintigraphy and heart rate variability. Eur J Nucl Med Mol Imaging 2010;37:1698-1705.

20 Lee SH, Bae HJ, Yoon BW, Kim H, Kim DE, Roh JK: Low concentration of serum total cholesterol is associated with multifocal signal loss lesions on gradient-echo magnetic resonance imaging: analysis of risk factors for multifocal signal loss lesions. Stroke 2002;33:2845-2849.

-21 Luchsinger JA: Type 2 diabetes, related conditions, in relation and dementia: an opportunity for prevention? J Alzheimers Dis 2010;20:723-736.

22 Reitz C, Tang MX, Manly J, Mayeux R, Luchsinger JA: Hypertension and the risk of mild cognitive impairment. Arch Neurol 2007;64:1734-1740.

-23 Soennesyn H, Oppedal K, Greve OJ, Fritze F, Auestad BH, Nore SP, Beyer MK, Aarsland D: White matter hyperintensities and the course of depressive symptoms in elderly people with mild dementia. Dement Geriatr Cogn Disord Extra 2012;2:97-111.

24 Orimo S, Ozawa E, Nakade S, Sugimoto T, Mizusawa H: (123)I-metaiodobenzylguanidine myocardial scintigraphy in Parkinson's disease. J Neurol Neurosurg Psychiatry 1999;67:189-194.

25 Snowdon DA, Greiner LH, Mortimer JA, Riley KP, Greiner PA, Markesbery WR: Brain infarction and the clinical expression of Alzheimer disease. The Nun Study. JAMA 1997;277:813-817. 